\title{
The Effects of Low Intensity Endurance Activity on Various Physiological Parameters and Exercise Induced Oxidative Stress in Dogs
}

\author{
Janice L. Huntingford1, Corri B. Levine², Debbie J. Mustacich³, Devin Corrigan3, \\ Robert L. Downey4, Joseph J. Wakshlag2* \\ ${ }^{1}$ Essex Animal Hospital, Essex, Canada \\ ${ }^{2}$ Department of Clinical Sciences, Cornell University, Ithaca, NY, USA \\ ${ }^{3}$ Linus Pauling Institute, Oregon State University, Corvallis, OR, USA \\ ${ }^{4}$ Annamaet Petfood Company, Sellersville, PA, USA \\ Email: jw37@cornell.edu
}

Received 8 June 2014; revised 8 July 2014; accepted 18 July 2014

Copyright (C) 2014 by authors and Scientific Research Publishing Inc.

This work is licensed under the Creative Commons Attribution International License (CC BY).

http://creativecommons.org/licenses/by/4.0/

(c) (i) Open Access

\begin{abstract}
The study of canine athlete physiology has focused on endurance sled dog racing and high intensity short duration Greyhound racing, yet the number of dogs competing in low intensity endurance activities is rising due to the increased numbers of hunting and companion dog activities. There is little information on the physiological effect of longer duration low intensity endurance activities. We set out to evaluate the serum biochemistry, oxidative stress, and cortisol response before and after two consecutive days of exercise in ten healthy unconditioned male dogs. Exercise sessions consisted of 120 minutes on an exercise wheel at $11 \mathrm{~km} /$ hour on 2 consecutive days. Blood was collected at four time points: 24 hours pre-exercise (sample 1, Day 0, resting); 2 min post-exercise on days 1 and 2, (samples 2 and 3, respectively); and 20 hours post-exercise, collected on day 3 (sample 4). Hematocrit, blood gases, serum chemistry, uric acid, cortisol, and $F_{2}$-isoprostanes were determined. Serum biochemistry and hematocrit suggested hemoconcentration, mild muscle damage and respiratory alkalosis during exercise, which was expected in the unconditioned canine athlete. In addition, plasma indices of oxidative damage ( $F_{2}$-isoprostanes) increased, as did plasma uric acid (an endogenous antioxidant). Importantly, similar to human studies, plasma $F_{2}-$ isoprostanes decreased 24 hours after exercise suggesting a protective effect of exercise. Serum cortisol concentrations were also markedly elevated at the end of exercise on both days suggesting that timing of sampling may play a role in interpreting cortisol results when looking at previous field studies.
\end{abstract}

"Corresponding author. 


\section{Keywords}

\section{Exercise, Cortisol, Isoprostane, Dehydration, Uric Acid}

\section{Introduction}

The study of exercise physiology in canine athletes has focused primarily on the endurance sled dog and the sprinting Greyhound [1]-[4]. However, in the last 10 years, the number of dogs competing in other canine sports has increased significantly. These include the low intensity endurance activities (30\% - 40\% $\mathrm{VO}_{2}$ max) typical amongst law enforcement, military, detection, search and rescue, recreational hunting dogs and companion dog "athletes". In addition, pet owners are also engaging in more physical activities with their canine companions including running, swimming, biking and skijoring (http://www.akc.org/about/annual_report.cfm). In many cases, unlike racing greyhounds and sled dogs, dogs participating in these low intensity endurance activities are not well conditioned and perform activities only on weekends or sporadically due to seasonality of the activities [5].

Presently, the only information on canine endurance exercise comes from the examination of the physiological extreme of the high intensity endurance activity of sled dog racing in highly conditioned athletes [2] [3] [6]. Depending on population and/or duration of activity, studies of physiological changes in sled dogs have suggested both hemoconcentration and hemodilution [3] [7] [8]. In addition, hydration status and kennel management have been shown to significantly affect hemodynamics in dogs within the same race [7].Considering all of these possible confounding variables present in many sled dog studies, it is not surprising that these studies have demonstrated significant inter-individual, as well as inter-study, variation in measures of exercise-induced muscle damage, i.e., plasma creatine kinase (CK) and aspartate amino-transferase (AST) [3] [6].

Limited field studies have been conducted on agility dogs, hunting foxhounds, Labrador retrievers, search and rescue dogs, and scenting dogs [9]-[12], and the current literature contains little information on longer duration low intensity exercise in the unconditioned canine. In addition, similar to sled dog studies, field studies of low intensity endurance activities are difficult to standardize and the inherent variability in exercise intensity makes interpretation difficult and may lead to contradictory inter-study results. Thus, we were interested to utilize additional measures of exercise-induced stress during low intensity endurance exercise using a controlled field setting for the exercise sessions.

Exhaustive exercise, i.e., human ultramarathon or endurance sled dog racing, increased production of free radicals (particularly oxygen-and nitrogen-centered radicals)leads to exercise-induced oxidative stress, including increased formation of $\mathrm{F}_{2}$-isoprostanes [13] [14]. Four groups of $\mathrm{F}_{2}$-isoprostane regioisomers (5-, 8-, 12-, and 15-series $\mathrm{F}_{2}$-isoprostanes) can be formed during free-radical mediated oxidation of arachidonic acid. The 15-series $\mathrm{F}_{2}$-isoprostanes (15R- and 15S-8-isoprostanes (8-isoPs))are the most extensively studied and are considered a biomarker of choice to assess in vivo oxidative stress, particularly when measured by either GC/MS or LC/MS[15] [16]. In studies using a commercial ELISA method for measurement of 8-isoPs, endurance sled dogs show 3- to 5 -fold increase in plasma $\mathrm{F}_{2}$-isoprostanes after running $100 \mathrm{~km}$, while agility dogs during competition showed no increase in $\mathrm{F}_{2}$-isoprostanes [12] [14]. These discrepancies may be genuine or may be the result of cross-reactivity to other compounds by the polyclonal antibody used in these commercial ELISA assays [15]. Importantly, 8-isoP levels have not been determined during exercise activities falling between these two extremes, i.e., during the more common low intensity longer duration activities of the unconditioned canine companion athlete. Interestingly, in contrast to exhaustive exercise, moderate intensity exercise in humans results in an adaptive response whereby markers of oxidative stress are significantly increased after the initial bout of moderate exercise, but become progressively lower after repeated bouts of moderate exercise[17]-[19]. Similar studies in canines are unavailable.

Uric acid, a potent antioxidant, is suggested to prevent elevations in oxidative damage [14] [20]. Elevated serum uric acid during both short and long duration strenuous activities is associated with decreased exercise-induced oxidative damage [20]-[22]. In addition, supplementation of humans with uric acid prevented exercise-induced elevation of 8-isoPs [23]. To date, similar studies have not been undertaken during low intensity endurance activities in either conditioned or unconditioned dogs.

Similar to exercise-induced oxidative stress, measures of the exercise-induced physiological stress have not 
been reported in dogs immediately following low intensity endurance activity. Physiologic stress is commonly assessed through serum cortisol as an indication of the activation of the adreno-hypothalamus axis. Cortisol has been shown to be elevated during an array of athletic and non-athletic canine activities [24]-[26], but the data varies with the activity. Sled dogs running between 10 and 120 minutes exhibited a marked rise in serum cortisol, while cortisol concentrations did not increase in agility dogs during short duration exercise [7] [11] [24]. These differences may be a reflection of the timing of sampling as well as variation in exercise, excitement and breed. To date, $\mathrm{F}_{2}$-isoprostane and uric acid levels, as well as markers of muscle damage, hemodynamics and cortisol, have not been determined during low intensity endurance activities in unconditioned dogs.

The purpose of this study was to assess biochemical and physiological parameters in unconditioned dogs during repeated low intensity endurance activity (30\% - 40\% $\mathrm{VO}_{2}$ max)in a controlled field environment by measuring clinical biomarkers of exercise-induced stress (serum chemistry and cortisol), as well as uric acid and plasma $\mathrm{F}_{2}$-isoprostanes.

\section{Materials and Methods}

\subsection{Dog Exercise Sessions}

All procedures in this study were approved by the Cornell University institutional care and use committee and owner consent was acquired for the study.

Ten healthy, unconditioned, male mixed breed dogs from a single kennel between the ages of 6 - 9 years of age weighing between 24 and 27 kilograms were used in this study. All of the dogs in the study were fed a commercial kibble containing 26\% crude protein and 16\% crude fat (Annamaet Extra, Annamaet Pet food, Sellersville, PA).Blood was collected at four different time points: a pre-exercise resting sample 24 hours before the first exercise session (day 0); a post-run sample on day 1 within 2 minutes of cessation of exercise (day 1); a post-run sample on day 2 within 2 minutes of cessation of exercise (day 2); and a sample 24 hours after the cessation of the day 2 exercise session (day 4). Exercise consisted of all dogs running simultaneously on an exercise wheel as is typical for conditioning canine athletes (sled dogs and hunting dogs) at $11 \mathrm{~km} /$ hour for 120 minutes on two consecutive days between 7 and 9 am. This exercise regimen simulates a low intensity endurance exercise equivalent to approximately $30 \%-40 \%$ of their maximal oxygen consumption [20]. At each half hour interval of exercise, the direction in which the dogs ran was changed. Immediately after exercise (within 60 seconds) venipuncture of the jugular vein was performed using a 20-gauge needle on each dog and 10 milliliters of blood was collected.1-ml of blood was transferred to a $1 \mathrm{ml}$ lithium heparin tube, 5-ml to a coagulation tube and $4 \mathrm{ml}$ to another lithium heparin tube. Serum and plasma were separated within 20 minutes of collection by centrifugation of the respective tubes for 6 minutes at $4000 \times \mathrm{g}$, transferred to separate tubes, immediately frozen on dry ice, and placed in liquid nitrogen for transportation to the laboratory. After transportation to the respective laboratories the samples were stored at $-80^{\circ} \mathrm{C}$ until analysis.

\subsection{Hematocrit}

Heparinized hematocrit capillary tubes were filled for each dog at each time point and spun on a hematocrit centrifuge.

\subsection{Serum Chemistry}

Serum chemistry was determined using an Olympus AU5400 automated analyzer (Olympus America, Center Valley, PA, USA).

\subsection{Blood Gas, Lactate, Uric Acid and Cortisol}

Blood gas and serum lactate was determined using an iSTAT CG8+ cartridge per the manufacturer (Abbott Laboratories, Deerfield IL). The cartridge was filled with heparinized blood within 5 minutes of blood draw. Cortisol was determined utilizing a validated radioimmunoassay for dogs (Cornell University Diagnostic Laboratory, Ithaca, NY). Uric acid was determined utilizing a commercial ELISA kit per manufacturer's instructions (Cay- 
man Chemical, Anna Arbor, MI).

\subsection{Plasma F2-Isoprostanes}

Plasma samples were shipped to the Linus Pauling Institute at Oregon State University for $\mathrm{F}_{2}$-isoprostane analysis. Plasma 15-series F2-isoprostanes (15R- and 15S-8-isoprostanes, 8-isoPs) and 5-series $\mathrm{F}_{2}$-isoprostane metabolites were extracted and measured by LC/MS as described by Taylor et al. [27]. Briefly, plasma was extracted by SPE as described [28] using a Strata-X SPE cartridge (500 mg/3 mL, Phenomenex, Torrance, CA). Eluted samples were dried under nitrogen and reconstituted with $200 \mu \mathrm{L}$ of methanol containing $0.1 \%$ formic acid and stored at $-80^{\circ} \mathrm{C}$ until analysis. Samples were analyzed using an HPLC coupled to a triple-quadrupole mass spectrometer operated in negative mode. 5- and 15-series $\mathrm{F}_{2}$-isoprostanes were quantified based on an internal standard [27].

\subsection{Statistical Analysis}

Data was analyzed by one-way ANOVA using Prism (Graphpad, La Jolla, CA). Any data that was not normally distributed based on Shapiro Wilks testing was transformed prior to analysis. Tukey’s Post hoc tests were performed when overall time point effects were found to be significant. Comparisons between groups with $\mathrm{p}$ values $<0.05$ were considered significant. All data for Figures 1-3 (cortisol, uric acid and isoprostanes) are expressed at mean \pm standard error of the mean.

\section{Results}

Unconditioned dogs participated in 2 low intensity endurance exercise sessions on 2 consecutive days (days 1 and 2). Measures of physiologic and oxidative stress were determined $24 \mathrm{~h}$ pre-exercise (day 0), 2 min post-exercise (days 1 and 2), and 20 h post-exercise (day 3).

\subsection{Hematocrit and Serum Chemistry}

Compared to pre-exercise levels serum total protein, albumin, sodium, and chloride, as well as hematocrit and total iron binding capacity, increased immediately following each low intensity endurance exercise session and returned to pre-exercise levels on day 3 ( $p<0.05$; Table 1$)$. In contrast, immediately following the day 1 and day 2 low intensity endurance exercise sessions, phosphorus levels decreased to 65\% and 60\%, respectively, compared to pre-exercise levels ( $p<0.05$; Table 1). Phosphorus levels remained decreased $20 \mathrm{~h}$ post-exercise. Blood urea nitrogen (BUN) levels increased 6\% following day 2 exercise and returned to pre-exercise resting levels on day 3 ( $\mathrm{p}<0.05$ ). Glucose and potassium levels decreased to approximately $90 \%$ of pre-exercise levels following day 2 exercise, but returned to pre-exercise levels by day 3 (p $<0.05$; Table 1). Blood calcium levels decreased $3 \%$ following the $2^{\text {nd }}$ day of low intensity endurance exercise, compared to pre-exercise levels, and recovered by 20 h post-exercise $(p<0.05)$. Alkaline phosphatase levels was unaltered following the day 1 low intensity endurance exercise, but increased 25\% following low intensity endurance exercise on day 2 and remained elevated 20 h post-exercise, compared to pre-exercise levels (p < 0.05; Table 1). Importantly, following low intensity endurance exercise on day 1, AST and CK levels increased 1.8- and 2.5-fold, respectively, compared to pre-exercise levels (28 $\pm 10 \mathrm{U} / \mathrm{L}$ and $96 \pm 17 \mathrm{U} / \mathrm{L}$, respectively). Following the $2^{\text {nd }}$ low intensity endurance exercise session (day 2), AST and CK levels further increased to 2.4- and 4.6-fold pre-exercise levels ( $<0.05$; Table 1). AST, but not CK, levels returned to pre-exercise levels $20 \mathrm{~h}$ post-exercise $(p<0.05)$. Finally, iron and iron saturation increased approximately 2-fold following each low intensity endurance exercise session, compared to pre-exercise levels $(p<0.05)$ and both indices remained elevated 20 h post-exercise. Cholesterol, creatinine, magnesium, and ALT levels were unchanged during this study.

\subsection{Blood Gas and Lactate}

Blood gas parameters were determined pre-exercise and immediately following low intensity endurance exercise on days 1 and 2. Base Excess (1.7- and 1.7-fold), pH (0.5\% and 1.4\%), and lactate (2.3- and 2.0-fold) levels increased following each exercise session compared to pre-exercise levels (Table 2, $\mathrm{p}<0.05$ ). Total $\mathrm{CO}_{2,} \mathrm{pCO}_{2}$, 
Table 1. Hematological and serum chemistry parameters in dogs at rest on day 0 , after exercise on day 1 and day 2 , and on day 320 hrs post-exercise. Data are expressed as mean \pm standard deviations. ${ }^{*}=p<0.05$ compared to pre-exercise levels (resting).

\begin{tabular}{|c|c|c|c|c|}
\hline Serum Chemistry & Day 0 (Rest) & Day 1 Ex. & Day 2 Ex. & Day 320 hr Post \\
\hline Total protein $(5.0-7.4 \mathrm{~g} / \mathrm{dL})$ & $6.1 \pm 0.3$ & $6.5 \pm 0.2^{*}$ & $6.4 \pm 0.3^{*}$ & $6.1 \pm 0.3$ \\
\hline Albumin (2.7 - 4.4 g/dL) & $3.7 \pm 0.1$ & $3.9 \pm 0.1^{*}$ & $3.9 \pm 0.1^{*}$ & $3.7 \pm 0.2$ \\
\hline Globulin $(1.6$ - $3.6 \mathrm{~g} / \mathrm{dL})$ & $2.4 \pm 0.3$ & $2.6 \pm 0.1$ & $2.5 \pm 0.2$ & $2.4 \pm 0.2$ \\
\hline AST (SGOT) (15 - 66 U/L) & $28 \pm 10$ & $51 \pm 12^{*}$ & $68 \pm 17^{*}$ & $42 \pm 16$ \\
\hline ALT (SGPT) (12 - 118 U/L) & $90 \pm 47$ & $99 \pm 48$ & $107 \pm 58$ & $99 \pm 50$ \\
\hline Alkaline phos. (5 - 131 U/L) & $52 \pm 17$ & $53 \pm 18$ & $65 \pm 26^{*}$ & $70 \pm 22^{*}$ \\
\hline Urea Nitrogen (6 - 31mg/dL) & $15 \pm 3$ & $15 \pm 2$ & $16 \pm 3^{*}$ & $12 \pm 3$ \\
\hline Creatinine $(0.5$ - $1.6 \mathrm{mg} / \mathrm{dL})$ & $0.9 \pm 0.1$ & $0.9 \pm 0.1$ & $1.0 \pm 0.2$ & $0.8 \pm 0.1$ \\
\hline Phosphorus (2.5 - 6.0 mg/dL) & $4.4 \pm 0.5$ & $2.9 \pm 0.8^{*}$ & $2.7 \pm 0.8^{*}$ & $3.5 \pm 0.5^{*}$ \\
\hline Glucose (70 - 138 mg/dL) & $96 \pm 6$ & $93 \pm 9$ & $86 \pm 9^{*}$ & $98 \pm 10$ \\
\hline Calcium (8.9 - 11.4 mg/dL) & $10.2 \pm 0.2$ & $10.2 \pm 0.2$ & $9.9 \pm 0.3^{*}$ & $10.1 \pm 0.2$ \\
\hline Magnesium (1.5 - $2.5 \mathrm{mEq} / \mathrm{L})$ & $1.7 \pm 0.1$ & $1.7 \pm 0.1$ & $1.7 \pm 0.1$ & $1.7 \pm 0.1$ \\
\hline Sodium (139 - $154 \mathrm{mEq} / \mathrm{L})$ & $148 \pm 1$ & $153 \pm 1^{*}$ & $151 \pm 3^{*}$ & $146 \pm 2$ \\
\hline Potassium (3.6 - $5.5 \mathrm{mEq} / \mathrm{L})$ & $4.9 \pm 0.2$ & $4.7 \pm 0.1$ & $4.5 \pm 0.3^{*}$ & $4.9 \pm 0.2$ \\
\hline Chloride (102 - 120 mEq/L) & $115 \pm 2$ & $118 \pm 2^{*}$ & $117 \pm 2^{*}$ & $114 \pm 2$ \\
\hline Cholesterol (92 - 324 mg/dL) & $179 \pm 37$ & $186 \pm 36$ & $187 \pm 37$ & $186 \pm 40$ \\
\hline Creatine Kinase (59 - 895 U/L) & $96 \pm 17$ & $216 \pm 60^{*}$ & $440 \pm 147^{*}$ & $196 \pm 108^{*}$ \\
\hline Hematocrit \% (40 - 56) & $51 \pm 3$ & $55 \pm 2^{*}$ & $55 \pm 4^{*}$ & $52 \pm 2$ \\
\hline Iron (ug/dL) & $98 \pm 15$ & $210 \pm 39^{*}$ & $200 \pm 41^{*}$ & $126 \pm 20^{*}$ \\
\hline Iron Saturation\% & $31.7 \pm 5.4$ & $64.4 \pm 13.9^{*}$ & $61.8 \pm 11.3^{*}$ & $40.9 \pm 8.5^{*}$ \\
\hline TIBC (ug/dL) & $310 \pm 36$ & $328 \pm 34^{*}$ & $327 \pm 43^{*}$ & $311 \pm 38$ \\
\hline
\end{tabular}

Table 2. Blood gas parameters in dogs at rest on day 0 , after exercise on day 1 and day 2. Data are expressed as mean \pm standard deviations. ${ }^{*}=\mathrm{p}<0.05$ compared to pre-exercise levels (resting).

\begin{tabular}{cccc}
\hline & Day 1 Rest & Day 1 Exercise & Day 2 Exercise \\
\hline Base Excess $(-2-+3 \mathrm{mmol} / \mathrm{L})$ & $-3 \pm 1$ & $-5 \pm 2^{*}$ & $-5 \pm 2^{*}$ \\
$\mathrm{pH}(7.31-7.41)$ & $7.40 \pm 0.21$ & $7.44 \pm 0.15^{*}$ & $7.50 \pm 0.10^{*}$ \\
$\mathrm{HCO}_{3}(23-28 \mathrm{mmol} / \mathrm{L})$ & $22 \pm 2$ & $19 \pm 2^{*}$ & $19 \pm 2^{*}$ \\
$\mathrm{TCO}_{2}(24-29 \mathrm{mmol} / \mathrm{L})$ & $23 \pm 2$ & $20 \pm 2^{*}$ & $19 \pm 2^{*}$ \\
$\mathrm{PCO}(41-51 \mathrm{mmHg})$ & $36 \pm 4$ & $29 \pm 5^{*}$ & $26 \pm 4^{*}$ \\
Lactate $(0.9-1.7 \mathrm{mmol} / \mathrm{L})$ & $0.7 \pm 0.3$ & $1.6 \pm 0.9^{*}$ & $1.4 \pm 1.8^{*}$ \\
\hline
\end{tabular}

and bicarbonate levels decreased following each exercise session (Table 2, p < 0.05).

\subsection{Cortisol and Uric Acid}

Serum cortisol concentrations increased 5.6- and 5.1-fold following low intensity endurance exercise on days 1 
and 2, compared to pre-exercise levels $(1.9 \pm 0.3 \mathrm{ug} / \mathrm{mL}, \mathrm{p}<0.01)$, but returned to pre-exercise levels by day 3 (Figure 1). Following the low intensity endurance exercise session on day 1 serum uric acid levels were increased more than 2-fold compared to pre-exercise levels (59.6 $55.2 \mu \mathrm{M}$ vs. $26.9 \pm 1.8 \mu \mathrm{M}$, p < 0.01) (Figure 2). Serum uric acid levels were also increased $(47.7 \pm 4.2 \mu \mathrm{M})$ following day 2 exercise, compared to pre-exercise levels ( $<0.05$ ), but recovered by 20 h post-exercise $(32.6 \pm 2.8 \mu \mathrm{M})$ (Figure 2 ).

\section{4. $\mathrm{F}_{2}$-Isoprostanes}

Due to the inter-individual variation (range 384 - 744 ng/mL 15-series $\mathrm{F}_{2}$-isoprostane; 954 - 1893 ng/mL 15 series- $\mathrm{F}_{2}$-isoprostanes) for pre-exercise $\mathrm{F}_{2}$-isoprostane levels, day 1,2 and 3 plasma $\mathrm{F}_{2}$-isoprostane levels are expressed as percent of pre-exercise levels, i.e., \%day 0. Immediately following low intensity endurance exercise on day 1, 15-series $\mathrm{F}_{2}$-isoprostane (8-IsoPs) levels increased 20\%, compared to resting levels (p < 0.01). However, following the low intensity endurance exercise session on day 2, 15-series $\mathrm{F}_{2}$-isoprostanelevels did not differ from resting levels. Interestingly, plasma 8-IsoPs levels were 77\% of resting levels by 20 h post-exercise $\left(p<0.05\right.$; Figure 3(a)). Although the 5-series $\mathrm{F}_{2}$-isoprostane levels show an increasing trend following low intensity endurance exercise on day 1 , this increase did not reach significance. Similarly to the 8-IsoPs, 5-series $\mathrm{F}_{2}$-isoprostane levels were decreased $20 \mathrm{~h}$ post-exercise (day 3 ) compared to pre-exercise levels ( $\mathrm{p}<0.05$; Figure $3(\mathbf{b})$ ).

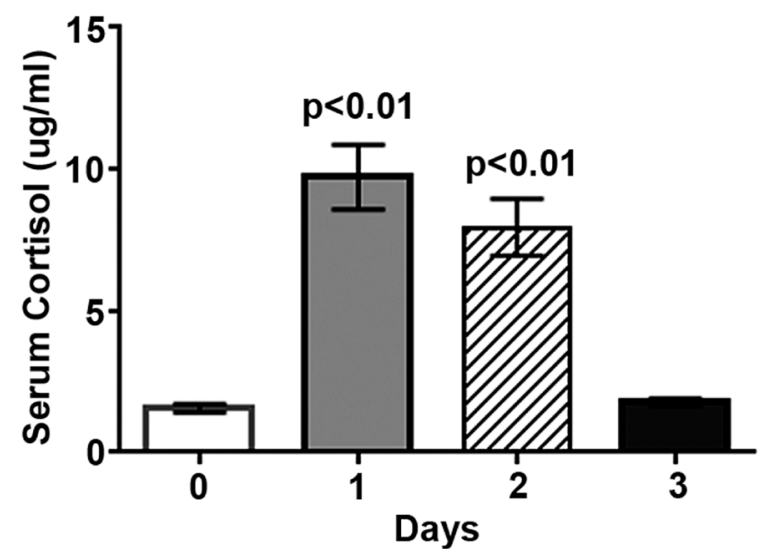

Figure 1. Low intensity endurance exercise increases serum cortisol. Unconditioned dogs participated in 2 low intensity endurance exercise sessions on 2 consecutive days (days 1 and 2) followed by a day of rest. Serum cortisol was determined 24 h pre-exercise (day 0, resting), 2 min post-exercise (days 1 and 2), and 20 h post-exercise (day 3). Data are expressed as mean \pm SEM, $n=10 ; p<0.01$ compared to pre-exercise (day 0 ).

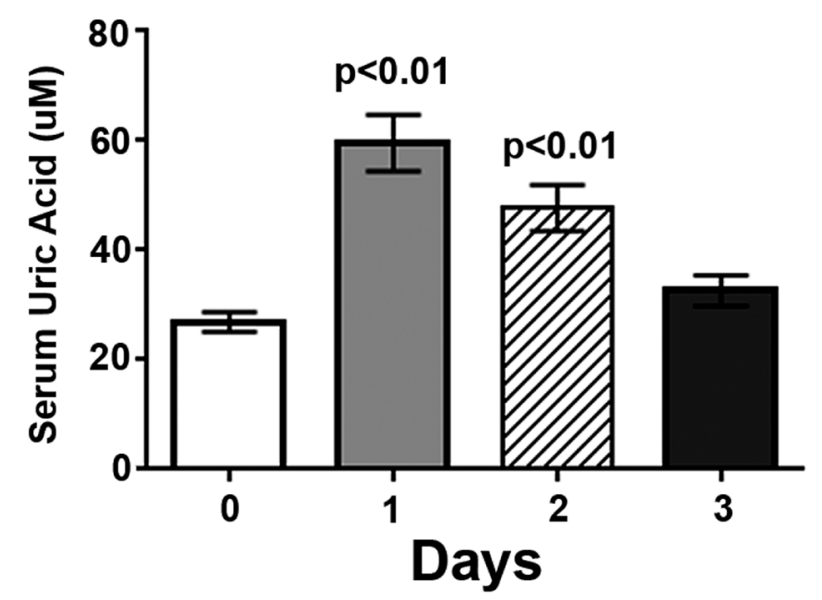

Figure 2. Low intensity endurance exercise increases serum uric acid levels. Unconditioned dogs participated in 2 low intensity endurance exercise sessions and serum was collected as described in Figure 1 and Methods. Data are expressed as mean \pm SEM, $\mathrm{n}=10 ; \mathrm{p}<0.01$ compared to pre-exercise (day 0 ). 


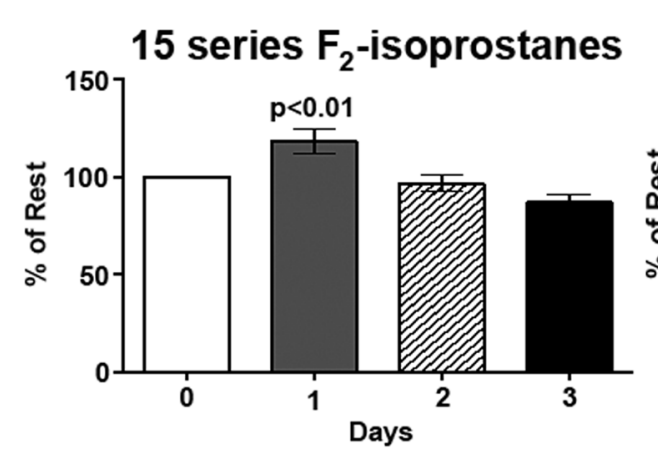

(a)

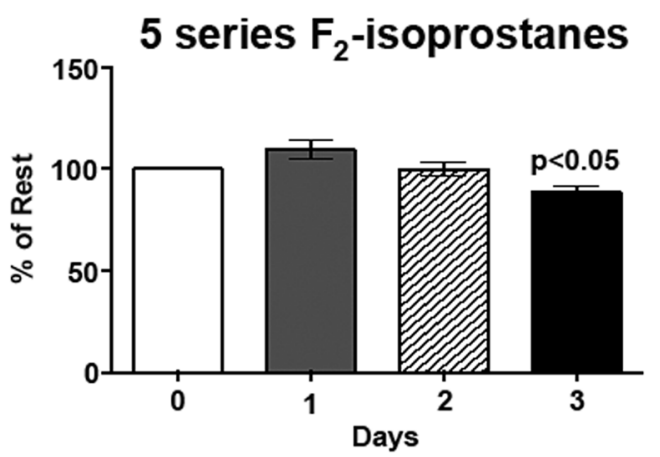

(b)

Figure 3. Low intensity endurance exercise alters plasma 15- and 5-series $\mathrm{F}_{2}$-isoprostane levels. Unconditioned dogs participated in 2 low intensity endurance exercise sessions on 2 consecutive days (days 1 and 2) followed by a day of rest. $\mathrm{F}_{2}$-isoprostanes were determined $24 \mathrm{~h}$ pre-exercise (day 0), 2 min post-exercise (days 1 and 2 ), and $20 \mathrm{~h}$ post-exercise (day 3 ) as described in Methods. a) 15-series $\mathrm{F}_{2}$-isoprostane levels and b) 5-series $\mathrm{F}_{2}$-isoprostane levels. Data are expressed as mean \pm SEM, $n=10$. Comparisons with p values $<0.05$ are considered significant as compared to pre exercise (day 0 ).

\section{Discussion}

To date, canine athlete physiology studies have focused primarily on endurance sled dog racing and high intensity short duration Greyhound racing. However, the number of dogs competing in other canine activities has risen significantly over the past 10 years, particularly low intensity endurance activities. Presently there is a lack of information on the physiological effect of low intensity endurance activities in canines, particularly in the unconditioned dog. In the current study, we determined serum biochemistry, oxidative stress, and cortisol response before and after two consecutive days of controlled low intensity exercise on an exercise wheel, as well as $20 \mathrm{~h}$ post-exercise, in ten healthy unconditioned male dogs. Interestingly, although $\mathrm{F}_{2}$-ioprostanes increased modestly following the day 1 exercise session, $20 \mathrm{~h}$ following the day 2 exercise session these indicators of oxidative stress were decreased below day 0 pre-exercise levels. These data suggest an adaptive response to repeated moderate exercise that may be beneficial to the long-term health of canines.

Although more consistent with physiologic changes seen in hunting or agility dogs, low intensity endurance exercise produced a unique set of biochemical changes compared to either hunting and agility dogs [1] [4] [9] [29] or endurance sled dogs [2]-[4]. Similar to agility dogs following competition, dogs in our study showed increased HCT, total protein, sodium, alkaline phosphatase, AST, and CK, but not ALT [11]. In addition, in contrast to agility dogs, our dogs demonstrated decreased serum phosphorus and glucose levels, which may have been a reflection of diet and/or energetics and phosphorus utilization in unconditioned dogs. Modest drops in phosphorus are common, reflecting the need for negative ions to move intracellularly due to respiratory alkalosis and increased glucose utilization in skeletal muscle requiring more intracellular phosphorus [9] [30] [31].

Following an endurance sled dog race changes in serum biochemistry were opposite compared to those seen following low intensity endurance exercise, i.e., decreased albumin, total protein and sodium, and increased phosphorus. The only similarity between the findings in sled dogs and our study dogs was increased chloride and decreased potassium. Elevations in HCT, total protein, albumin, sodium, chloride and blood urea nitrogen can be explained in part by dehydration and inter compartmental fluid shifts causing hemoconcentration [1] [3]. Sympathetic induced splenic contraction occurs in agility dogs and Greyhounds and is thought to partially explain elevations in hematocrit post exercise [1] [29]. However, our increases were mild compared to those observed in Greyhounds. Thus it would appear that both of these processes occurred in the study dogs with complete recovery 20 hours post-exercise.

Immediately following low intensity endurance exercise, the serum enzymes CK and AST increased significantly indicating that exercise induced damage to the muscle cell membrane had occurred [2] [7] [32]. CK did not recover within 20 hours, likely due to continual rise that occurs after the cessation of exercise for up to 12 hours [33]. Lactate has been shown to be an indicator of glycolytic activity in skeletal muscle and the magnitude of the rise is related to intensity of exercise and fitness of the individual. Lactate was elevated on day 1 and day 
2 of the exercise, however the rises were still within the reference interval. When compared to Greyhounds and agility dogs who show a 3- to 10-fold higher serum lactate concentration after an exercise bout, our study dogs showed little muscle glycolytic activity after 2 hours of low intensity exercise [12] [34]. This suggests that the dogs were running primarily from energy generated from the beta-oxidation of fatty acids, which would be expected for dogs running between $30 \%-50 \%$ of their maximal oxygen capacity [20] [35]. However, even at this low intensity of exercise, our study dogs appeared to be alkalotic, similar to field trial Labradors who showed a pronounced respiratory alkalosis following exercise [36]. This is expected since panting is the major thermoregulatory mechanism for the dog. Panting causes increased loss of $\mathrm{CO}_{2}$ through respiration resulting in a mild alkalosis that recovers quickly in dogs [1] [36]. This drop in serum bicarbonate and modest decrease in phosphorus may have led to the mild hyperchloremia observed in our dogs. These changes may represent an effort to replace intravascular negative ions for acid base balance [8] [30].

Uric acid is a constantly formed metabolite of purine metabolism in all mammals, and also a potent endogenous antioxidant that acts in concert with ascorbic acid [37] [38]. Typically, uric acid concentrations do not rise dramatically unless purines are being metabolized from diet or from endogenous sources into carbon moieties for other metabolic purposes [37]. However, uric acid levels have been shown to increase following endurance exercise in both dogs and humans. Specifically, uric acid levels increased 2-fold in endurance sled dogs running 30 minutes at high intensity $\left(70 \% \mathrm{VO}_{2}\right.$ max) and were increased as much as 3-fold in sled dogs after running 100 miles [20]-[22]. Increased uric acid levels have been proposed as a protective mechanism against exercise-induced oxidative stress [20] [22]. The 2- to 3-fold increase following low intensity endurance activity suggests that this protective mechanism may be in place during most exercise of any intensity or duration and should be examined in sprint athletes.

Similar to racing sled dogs, plasma $\mathrm{F}_{2}$-isoprostane levels increased immediately following the first bout of exercise, compared to resting levels. However, $F_{2}$-isoprostane levels in our study dogs increased only $20 \%$ while levels in the racing sled dogs increased more than 3-fold [14]. In addition, while $\mathrm{F}_{2}$-isoprostane levels remained elevated following the third day of the sled dog race, day $2 \mathrm{~F}_{2}$-isoprostane levels did not differ from resting levels in our study dogs. Furthermore, in our study, $20 \mathrm{~h}$ post-exercise $\mathrm{F}_{2}$-isoprostane levels were decreased below resting levels. Our data are in contrast with data reported by Dunlap and colleagues [20] in which rises in uric acid were coupled with non-significant elevations in plasma $F_{2}$-isoprostane levels. Additionally, Baltzer and colleagues [12] showed no differences in urinary or serum $\mathrm{F}_{2}$-isoprostane levels in agility dogs after exercise. These differences may be due to the higher specificity and sensitivity of the mass spectroscopy method used in our current study compared to the ELISA method used by the earlier studies [15] [39]. ELISA based assays utilize antibodies that have shown significant cross reactivity with other compounds in addition to the 8- $\mathrm{F}_{2}$-isoprostane they are intended to measure [27]. Mass spectroscopy coupled with either gas chromatography or HPLC is the preferred analytical technique for in vivo measurement of $F_{2}$-isoprostanes [15] [39]. In addition, Dunlap indicates that the recommended sample extraction was not done in their study and this may further explain why increases did not reach significance in that study [20]. Importantly, our data shows that 20 hours after 2 consecutive days of low intensity exercise $\mathrm{F}_{2}$-isoprostane levels decreased significantly suggesting a protective effect of exercise, as previously observed in human studies of moderate exercising [17] [19].

Finally, our data demonstrates a dramatic rise in serum cortisol in unconditioned dogs that was similar to, but considerably more robust than studies of endurance sled dogs, sprinting sled dogs, field trial or agility dogs [3] [6] [11] [12] [32]. In studies of endurance and sprinting huskies, blood draws occurred within 30 minutes of cessation of exercise, whereas the agility trial blood draw was within 2 minutes and again 4 hours after cessation of exercise [3] [7] [12]. Since the serum half-life of cortisol is approximately 30 minutes, it is entirely possible that the more elevated concentrations observed in our study were due to the timing of our blood draw, i.e., within 60 seconds of the discontinuation of exercise. Together these data point to the importance of venipuncture timing when planning studies and interpreting results [40] [41].

Another possibility for the differences in cortisol results is that the dogs used in our study were unconditioned. Thus there may be greater stress associated with beginning a new exercise regimen. Further studies are needed to determine cortisol responses in the conditioned versus unconditioned dog. Finally, there could have been excitement by the unconditioned dogs when they were allowed to discontinue this exercise session. Such a response would prompt sympathetic stimulation resulting in a burst of cortisol. However, this is doubtful based on behaviors observed by researchers during and after the exercise sessions. 


\section{Conclusion}

In conclusion, this study demonstrated that the unconditioned canine athlete undergoes physiological changes similar to those observed in other exercising canines. However, unlike the endurance sled dog, the unconditioned dog shows modest hemoconcentration and physiological changes that favor fat oxidation rather than glycolysis. The low intensity endurance exercise used in this study did not induce a level of oxidative stress indicative of significant muscle damage. Rather, levels of the endogenous antioxidant uric acid were increased. In addition, there was a significant decrease in resting levels of $\mathrm{F}_{2}$-isoprostanes within 2 days of beginning training. These data suggest that, like humans, moderate exercise leads to conditioning and an improved ability to respond to oxidative stress in canines. Finally, the oxidative indices and serum cortisol measurements in this study highlight that sampling times and methodology are absolutely critical when interpreting findings in field studies.

\section{References}

[1] Ilkiw, J.E., Davis, P.E. and Church, D.B. (1989) Hematologic, Biochemical, Blood-Gas and Acid Base Values in Greyhounds before and after Exercise. American Journal of Veterinary Research, 50, 583-586.

[2] Hinchcliff, K.W., Olson, J. and Crusberg, C. (1993) Serum Biochemical Changes in Dogs Competing in a Long Distance Sled Race. Journal of the American Veterinary Medical Association, 202, 401-409.

[3] Burr, J.R., Reinhart, G.A. and Swenson, R.A. (1997) Serum Biochemical Values in Sled Dogs before and after Competing in Long-Distance Races. Journal of the American Veterinary Medical Association, 211, 175-179.

[4] Rose, R.J. and Bloomberg, M.S. (1989) Responses to Sprint Exercise In the Greyhound: Effects on Haematology, Serum Chemistry Serum Chemistry and Muscle Metabolites. Research in Veterinary Science, 47, 212-218.

[5] Piccione, G., Casella, S., Panzera, M., Giannetto, C. and Fazio, F. (2012) Effect of Moderate Treadmill Exercise on Some Physiological Parameters in Untrained Beagle Dogs. Experimental Animals, 61, 511-515. http://dx.doi.org/10.1538/expanim.61.511

[6] Wakshlag, J.J., Stokol, T., Geske, S.M., Gregen, C.E., Angle, C.T. and Gillette, R.L. (2010) Evaluation of Exercise-Induced Changes in Concentrations of C-Reactive Protein and Serum Biochemical Values in Sled Dogs Completing a Long-Distance Endurance Race. American Journal of Veterinary Research, 71, 1207-1213. http://dx.doi.org/10.2460/ajvr.71.10.1207

[7] Wakshlag, J.J., Snedden, K. and Reynolds, A.J. (2004) Biochemical and Metabolic Changes Due to Exercise in Sprint-Racing Sled Dogs: Implications for Post-Exercise Carbohydrate Supplements and Hydration Management. Veterinary Therapeutics, 5, 52-59.

[8] McKenzie, E.C., Jose-Cunilleras, E., Hinchcliff, K.W., Holbrook, T.C., Royer, C., Payton, M.E., Williamson, K., Nelson, S., Willard, M.D. and Davis, M.S. (2007) Serum Chemistry Alterations in Alaskan Sled Dogs during Five Successive Days of Prolonged Endurance Exercise. Journal of the American Veterinary Medical Association, 230, 1486-1492. http://dx.doi.org/10.2460/javma.230.10.1486

[9] Davenport, G.M., Kelley, R.L. and Altom, E.K. (2001) Effect of Diet on Hunting Performance in English Pointers. Veterinary Therapeutics, 2, 10-23.

[10] Steiss, J., Ahmad, H.A. and Cooper, P. (2004) Physiologic Response in Healthy Labrador Retrievers during Field Trial Training and Competition. Journal of Veterinary Internal Medicine, 18, 147-151. http://dx.doi.org/10.1111/j.1939-1676.2004.tb00153.x

[11] Rovira, S., Munoz, A. and Benito, M. (2008) Effect of Exercise on Physiological, Blood and Endocrine Parameters in Search and Rescue Trained Dogs. Veterinary Medicine, 53, 333-346.

[12] Baltzer, W.I., Firshman, A.M., Stang, B., Warnock, J.J., Gorman, E. and McKenzie, E.C. (2012) The Effect of Agility Exercise on Eicosanoid Excretion, Oxidant Status, and Plasma Lactate in Dogs. BMC Veterinary Research, 8, 249-257. http://dx.doi.org/10.1186/1746-6148-8-249

[13] Mastaloudis, A., Leonard, S.W. and Traber, M.G. (2001) Oxidative Stress in Athletes during Extreme Endurance Exercise. Free Radical Biology Medicine, 31, 911-922. http://dx.doi.org/10.1016/S0891-5849(01)00667-0

[14] Hinchcliff, K.W., Reinhart, G.A. and DiSilvestro, R. (2000) Oxidant Stress in Sled Dogs Subjected to Repetitive Endurance Exercise. American Journal of Veterinary Research. 61, 512-517. http://dx.doi.org/10.2460/ajvr.2000.61.512

[15] Milne, G.L., Yin, H., Hardy, K.D., Davies, S.S. and Roberts, L.J. (2011) Isoprostane Generation and Function. Chemical Reviews, 111, 5973-5996. http://dx.doi.org/10.1021/cr200160h

[16] Dorjgochoo, T., Gao, Y., Chow, W., Shu, X., Yang, G., Cai, Q., Rothman, N., Cai, H., Li, H., Deng, X., Franke, A., Roberts, L.J., Milne, G., Zheng, W. and Dai, Qi. (2012) Major Metabolite of $F_{2}$-Isoprostane in Urine May Be a More Sensitive Biomarker of Oxidative Stress than Isoprostane Itself. The American Journal of Clinical Nutrition, 96, 
405-414. http://dx.doi.org/10.3945/ajcn.112.034918

[17] Packer, L., Cadenas, E. and Davies, K.J. (2008) Free Radicals and Exercise: An Introduction. Free Radical Biology Medicine, 44, 123-125. http://dx.doi.org/10.1016/j.freeradbiomed.2007.05.031

[18] Sachdev, S. and Davies K.J. (2008) Production, Detection, and Adaptive Responses to Free Radicals in Exercise. Free Radical Biology Medicine, 44, 215-223. http://dx.doi.org/10.1016/j.freeradbiomed.2007.07.019

[19] Gomez-Cabrera, M.C., Domenech, E. and Viña, J. (2008) Moderate Exercise Is an Antioxidant: Upregulation of Antioxidant Genes by Training. Free Radical Biology Medicine, 44, 126-131. http://dx.doi.org/10.1016/j.freeradbiomed.2007.02.001

[20] Dunlap, K.E., Reynolds, A.J. and Duffy, L.K. (2006) Total Antioxidant Power in Sled Dogs Supplemented with Blueberries and the Comparison of Blood Parameters Associated with Exercise. Comparative Biochemistry and Physiology-Part A, 143, 429-434. http://dx.doi.org/10.1016/j.cbpa.2005.09.007

[21] Baskin, C.R., Hinchcliff, K.W., Disilvestro, R.A., Reinhart, G.A., Hayek, M.G., Chew, B.P., Burr, J.R. and Swenson, R.A. (2000) Effects of Dietary Antioxidant Supplementation on Oxidative Damage and Resistance to Oxidative Damage during Prolonged Exercise in Sled Dogs. American Journal of Veterinary Research, 6, 886-889. http://dx.doi.org/10.2460/ajvr.2000.61.886

[22] Piercy, R.J., Hinchcliff, K.W. and DiSilvestro, R.A., (2000) Effect of Dietary Supplements Containing Antioxidants on Attenuation of Muscle Damage in Exercising Sled Dogs. American Journal of Veterinary Research, 61, 1438-1445. http://dx.doi.org/10.2460/ajvr.2000.61.1438

[23] Waring, W.S., Convery A., Mishra V., Shenkin A., Webb D.J. and Maxwell S.R. (2003)Uric Acid Reduces Exercise-Induced Oxidative Stress in Healthy Adults. Clinical Science (London), 105, 425-430. http://dx.doi.org/10.1042/CS20030149

[24] Angle, C.T., Wakshlag, J.J., Gillette, R.L., Stokol, T., Geske, S., Adkins, T.O. and Gregor, C. (2009) Hematologic, Serum Biochemical, and Cortisol Changes Associated with Anticipation of Exercise and Short Duration High-Intensity Exercise in Sled Dogs. Veterinary Clinical Pathology, 38, 370-374. http://dx.doi.org/10.1111/j.1939-165X.2009.00122.x

[25] Hennessy, M.B., Davis H.N. and Williams, M.T. (1997) Plasma Cortisol Levels of Dogs at a County Animal Shelter. Physiology \& Behavior, 62,485-490. http://dx.doi.org/10.1016/S0031-9384(97)80328-9

[26] Haverbeke, A., Diederich, C., Depiereux, E. and Giffroy, J.M. (2008) Cortisol and Behavioral Response of Working Dogs to Environmental Challenges. Physiology \& Behavior, 93, 59-67. http://dx.doi.org/10.1016/j.physbeh.2007.07.014

[27] Taylor, A.W., Bruno, R.S., Frei, B. and Traber, M.G. (2006) Benefits of Prolonged Gradient Separation for High-Performance Liquid Chromatography-Tandem Mass Spectrometry Quantitation of Plasma Total 15-Series $\mathrm{F}_{2}$-Isoprostanes. Analytical Biochemistry, 350, 41-51. http://dx.doi.org/10.1016/j.ab.2005.12.003

[28] Taylor, A.W. and Traber, M.G. (2010) Quantitation of Plasma Total 15-Series $F_{2}$-Isoprostanes by Sequential Solid Phase and Liquid-Liquid Extraction. Analytical Biochemistry, 396, 319-321. http://dx.doi.org/10.1016/j.ab.2009.09.042

[29] Rovira, S., Munoz, A. and Benito, M. (2007) Hematologic and Biochemical Changes during Canine Agility Competitions. Veterinary Clinical Pathology, 36, 30-35. http://dx.doi.org/10.1111/j.1939-165X.2007.tb00178.x

[30] Willard, M.D. and DiBartola, S. (2000) Disorders of Phosphorus. In: DiBartola, S., Ed., Fluid Therapy in Small Animal Practice, 2nd Edition, W.B. Saunders Company, New York, 163-174.

[31] Hammel, E.P., Kronfled, D.S., Ganjam, V.K. and Dunlap, H.L. (1977) Metabolic Responses to Exhaustive Exercise in Racing Sled Dogs Fed Diets Containing Medium, Low or Zero Carbohydrate. American Journal of Clinical Nutrition, 30, 409-418.

[32] Huntingford, J.L., Kirn, B., Cramer, K. and Wakshlag, J.J. (2014) Evaluation of a Performance-Enhancing Supplement in American Foxhounds during Eventing. Journal of Nutritional Science, in press.

[33] Aktas, M., Auguste, D., Lefebvre, H.P., Toutain, P.L. and Braun, J.P. (1993) Creatine Kinase in the Dog: A Review. Veterinary Research Communications, 17, 353-369. http://dx.doi.org/10.1007/BF01839386

[34] Snow, D.H., Harris, R.C. and Stuttard, E. (1988) Changes in Haematology and Plasma Biochemistry during Maximal Exercise in Greyhounds. Veterinary Record, 123, 487-489. http://dx.doi.org/10.1136/vr.123.19.487

[35] Reynolds, A.J., Reinhart, G.A., Carey, D.P., Simmerman, D.A., Frank, D.A. and Kallfelz, F.A. (1997) Effect of Protein Intake during Training on Biochemical and Performance Variables Is Sled Dogs. American Journal of Veterinary Research, 60, 789-799.

[36] Steiss, J.E. and Wright, J.C. (2008) Respiratory Alkalosis and Primary Hypocapnia in Labrador Retrievers Participating in Field Trials in High-Ambient-Temperature Conditions. American Journal of Veterinary Research, 69, 12621267. http://dx.doi.org/10.2460/ajvr.69.10.1262 
[37] Ruggiero, C., Cherubini, A., Ble, A., Bos, A.J.G., Maggio, M., Dixit, V.D., Lauretani, F., Bandinelli, S., Senin, U. and Ferrucci, L. (2006) Uric Acid and Inflammatory Markers. European Heart Journal, 27, 1174-1181.

[38] Child, R.B., Wilkinson, D.M., Fallowfield, J.L. and Donnelly, A.E. (1998) Elevated Serum Antioxidant Capacity and Plasma Malondialdehyde Concentration in Response to a Simulated Half-Marathon Run. Medicine and Science in Sports and Exercise, 30, 1603-1607.

[39] Roberts II, L.J. and Milne, G.L. (2009) Isoprostanes. Journal of Lipid Research, 50, S219-S223. http://dx.doi.org/10.1194/jlr.R800037-JLR200

[40] Ferlazzo, A., Medica, P., Cravana, C. and Fazzio, E. (2009) Endocrine Changes after Experimental Show Jumping. Comparative Exercise Physiology, 6, 59-66.

[41] Ferlazzo, A., Medica, P., Cravana, C. and Fazzio, E. (2012) Circulating $\beta$-Endorphin Adrenocorticotropin, and Cortisol Concentrations of Horses before and after Competitive Show Jumping with Different Fence Heights. Journal of Equine Veterinary Science, 32, 740-746. http://dx.doi.org/10.1016/j.jevs.2012.02.021 